\title{
SACHÊS ANTIMICROBIANOS EM PÓS-COLHEITA DE MANGA ${ }^{1}$
}

\author{
EBER ANTONIO ALVES MEDEIROS², NILDA DE FÁTIMA FERREIRA SOARES ${ }^{3}$, \\ TIAGO DE OLIVEIRA SALES POLITO ${ }^{4}$, MIRIANE MARIA DE SOUSA ${ }^{5}$, \\ DANIELLE FABÍOLA PEREIRA SILVA ${ }^{6}$
}

RESUMO - Objetivou-se com este trabalho desenvolver sachês incorporados com óleos essenciais avaliando as propriedades físico-químicas e microbiológicas de mangas armazenadas em saco de papel contendo estes sachês em seu interior. Os óleos essenciais de orégano (Origanum vulgaris) e capim-limão (Cymbopogon citratus) foram incorporados em saches, e sua atividade antimicrobiana foi testada nos fungos Colletotrichum gloeosporides, Lasiodiplodia theobromae, Xanthomonas campestris pv. mangiferae indica, Alternaria alternata. Frutos de manga 'Tommy Atkins' foram acondicionados individualmente em sacos de papel contendo em seu interior um sachê antimicrobiano e mantidos a $25{ }^{\circ} \mathrm{C} \pm 2{ }^{\circ} \mathrm{C}$ e UR $80 \% \pm 5 \%$ por nove dias. Os parâmetros cor da epiderme, firmeza de polpa, sólidos solúveis, acidez titulável e $\mathrm{pH}$ sofreram pouca influência dos óleos essenciais de orégano e capim limão, indicando que a presença do óleo essencial não altera as características físico-químicas da polpa de manga. Os sachês ativos incorporados com óleos essenciais de orégano e capim limão apresentaram controle no crescimento dos microrganismos testados, sendo o capim-limão mais eficiente, reduzindo em aproximadamente 2 ciclos Log a contagem de mesófilos aeróbios e fungos filamentosos e leveduras em relação ao tratamento controle empregado.

Termos para indexação: pós-colheita; embalagens ativas; óleos essenciais.

\section{ANTIMICROBIAL SACHETS POST-HARVEST MANGO FRUITS}

\begin{abstract}
The present work aimed to develop sachets incorporated with essential oils to be stored with mangoes inside paper bags, as well as to assess both its antimicrobial and physicochemical properties. Oregano (Origanum vulgaris) and lemongrass (Cymbopogon citratus) essential oils were incorporated into sachets and their antimicrobial activity was tested for the fungi Colletotrichum gloeosporides, Lasiodiplodia theobromae, Xanthomonas campestris pv. mangiferae indica, and Alternaria alternate. Tommy Atkins mangoes were individually wrapped in paper bags containing antimicrobial sachets and maintained at $25^{\circ} \mathrm{C}$ $\pm 2{ }^{\circ} \mathrm{C}$ e RH $80 \% \pm 5 \%$, for nine days. Both oregano and lemongrass essential oils were found to have little effect on mango skin color, firmness, soluble solids, titratable acidity and $\mathrm{pH}$, indicating that their presence does not alter the physicochemical characteristics of mango pulp. Active sachets incorporated with either oregano or lemongrass essential oils showed efficiency, reducing the growth of the tested fungi, whereas lemongrass was found to be more efficient by decreasing about $2 \log$ cycles both the mesophilic aerobes and molds and yeasts counts, when compared to the control treatment.
\end{abstract}

Index terms: postharvest; active packaging; essentials oils.

\footnotetext{
${ }^{1}$ Trabalho Sinfruit 066 - Simpósio Internacional de Fruticultura - Avanços na Fruticultura (17 a 21 Outubro)

${ }^{2}$ Eng. Agr.- Pós-Doutorando (Bolsista PNPD/CNPq) - Departamento de Tecnologia de Alimentos - Universidade Federal de Viçosa, Av. PH. Rolfs s/n, Viçosa-MG, Brazil - 36570-000. E-mail: ebermedeiros@yahoo.com.br

${ }^{3}$ Eng $^{\mathrm{a}}$. Alimentos, Prof ${ }^{\mathrm{a}}$ Adjunta - Departamento de Tecnologia de Alimentos - Universidade Federal de Viçosa, Av. PH. Rolfs s/n, Viçosa-MG, Brazil - 36570-000. E-mail: nfsoares@ufv.br. Autora para correspondência

${ }^{4}$ Biomédico - Mestrando - Departamento de Tecnologia de Alimentos - Universidade Federal de Viçosa, Av. PH. Rolfs s/n, Viçosa MG, Brazil - 36570-000. E-mail: thiagopolito@yahoo.com.br

${ }^{5}$ Estudante de Ciência e Tecnologia de Laticínios - Departamento de Tecnologia de Alimentos - Universidade Federal de Viçosa, Av. PH. Rolfs s/n, Viçosa-MG, Brazil - 36570-000. E-mail: mirianesousa@yahoo.com.br

${ }^{6} E_{n g}$. Agra ${ }^{a}$, DSc.- Pós-Doutoranda -Universidade Federal de Viçosa, Departamento de Fitotecnia 36570-000, Viçosa-MG, Brazil. E-mail: danieele@ufv.br
} 


\section{INTRODUÇÃO}

A Manga (Mangifera indica L.) é uma das mais populares frutas tropicais, com origem no sudeste asiático e introduzida no Brasil no século XVI, dando origem a diversas variedades cultivadas (CARVALHO et al., 2004).

A exportação de manga teve crescimento significativo nos últimos anos, sendo que o principal fator que influencia o baixo volume nas exportações desta fruta in natura é a baixa qualidade dos frutos produzidos, devido a problemas fitossanitários e pouco conhecimento da fisiologia pós-colheita (LUCENA et al., 2007).

Para reduzir estas perdas, provocadas por doenças, e para que essa cultura seja economicamente competitiva no mercado, é necessário que se busquem tecnologias de produção e eficientes técnicas de preservação pós-colheita. Nos últimos anos, várias técnicas de conservação pós-colheita têm sido usadas para garantir a preservação, o frescor e a alta qualidade dos alimentos, dentre as quais podem ser citadas a atmosfera controlada e a embalagem em atmosfera modificada combinadas com baixas temperaturas (ALMENAR et al., 2009).

Assim, novos métodos de preservação de alimentos, como as embalagens ativas, estão sendo estudados e desenvolvidos para manter a qualidade do produto com maior tempo de prateleira (LEE et al., 1998).

Alguns pesquisadores têm encontrado atividade antimicrobiana no óleo essencial de orégano (BAYDAR et al., 2004). O orégano (Origanum vulgare L.) pertence à família Lamiaceae, conhecido por suas propriedades terapêuticas, sendo usado na medicina tradicional em vários países. É muito usado na indústria agronômica, farmacêutica e cosmética, bem como erva na culinária, substâncias flavorizantes em produtos alimentícios, bebidas alcoólicas e perfumaria (SOUZA et al., 2007).

As embalagens antimicrobianas apresentam-se de várias formas, e a aplicação comercial mais conhecida é na forma de sachês antimicrobianos, contendo agentes voláteis que permitem o controle do desenvolvimento de microrganismos. Uma forma para reduzir o crescimento de microrganismos é o uso de óleos essenciais. Muitas pesquisas têm sugerido o uso destes compostos naturais como possíveis substitutos aos fungicidas comerciais, indicando que têm potencial de aplicação na incorporação em sachê colocado no interior das mesmas (LEE et al., 1998; ZIVANOVIC et al., 2005).

Objetivou-se com este trabalho desenvolver sachês incorporados com óleos essenciais avaliando as propriedades físico-químicas e microbiológicas de mangas armazenadas em saco de papel contendo esses sachês em seu interior.

\section{MATERIAL E MÉTODOS}

Frutos de manga 'Tommy Atkins' foram adquiridos no comércio varejista de Viçosa-MG, em maio de 2010. Os frutos foram levados para o Laboratório de Embalagem, no Departamento de Tecnologia de Alimentos, Centro de Ciências Tecnológicas - Universidade Federal de Viçosa, onde foram selecionados quanto ao estádio de maturação e ausência de danos mecânicos.

Resina polimérica de alta absorção e tecido-não-tecido foram utilizados na confecção dos sachês, nos quais foram incorporados os óleos essenciais de orégano (Origanum vulgaris), e capim-limão (Cymbopogon citratus).

O delineamento experimental foi inteiramente casualizado (DIC), com cinco repetições. Cada tratamento foi composto de cinco frutos, sendo cada fruto considerado uma unidade experimental. Para as análises microbiológicas, utilizou-se de três repetições.

Os óleos essenciais foram avaliados para testar sua capacidade antimicrobiana pelo método de difusão em Ágar (APPENDINI; HOTCHKISS, 2002); , o qual consiste em discos de papel-filtro com $10 \mathrm{~mm}$ de diâmetro impregnados com $60 \mu 1$ de óleo e colocados no centro de uma placa de Petri, com meio BDA previamente inoculado com a suspensão de $0,1 \mathrm{~mL}$ de esporos de cada um dos fungos Colletotrichum gloeosporides, Lasiodiplodia theobromae, Xanthomonas campestris pv. mangiferae indica, Alternaria alternata. Em seguida, as placas de Petri foram incubadas a $35^{\circ} \mathrm{C}$ por 5 dias. A atividade dos óleos foi determinada a partir do diâmetro do halo de inibição formado ao redor do disco de papel. O delineamento experimental foi inteiramente casualizado (DIC), com três repetições em triplicata.

Os sachês ativos antimicrobianos foram elaborados a partir da incorporação dos OEs acima citados em $1 \mathrm{~g}$ de resina polimérica de alta absorção (Accurel ${ }^{\circledR}$ ), na concentração de $20 \%$ em relação à quantidade de resina. Após incorporação, a resina foi acondicionada em saquinhos de tecido-não-tecido (TNT).

Para a avaliação da eficiência dos sachês ativos antimicrobianos, eles foram fixados na face inferior da tampa das placas de Petri contendo ágar inoculado com $0,1 \mathrm{~mL}$ de esporos do microrganismo-teste. Após cinco dias, foi avaliada a presença ou ausência de unidades formadoras de colônias, e o 
resultado, expresso em $\mathrm{UFCmL}^{-1}$. Os microrganismos utilizados nos testes in vitro para a eficiência antimicrobiana dos sachês foram os mesmos utilizados para o teste de halo de inibição.

Frutos de manga foram acondicionados individualmente em sacos de papel contendo em seu interior um sachê antimicrobiano. Os óleos essenciais utilizados foram aqueles que apresentaram melhor controle do desenvolvimento de microrganismos durante o teste de halo de inibição in vitroDurante o período de conservação, os frutos foram mantidos a $25^{\circ} \mathrm{C} \pm 2{ }^{\circ} \mathrm{C}$ e UR $80 \% \pm 5 \%$, no laboratório de embalagens, simulando as condições a que são expostos no comércio varejista. Os parâmetros físico-químicos e microbiológicos foram avaliados a cada três dias, durante o período de conservação.

A cor da epiderme foi determinada em colorímetro (ColorQuest XE), com leitura direta de reflectância, no padrão C.I.E. L*a*b*. Para cada amostra, foi utilizada a média de cinco leituras em pontos diferentes da região equatorial dos frutos.

A firmeza de polpa foi determinada em texturômetro INSTRON, Série 3367. Duas leituras foram tomadas em lados opostos na região equatorial dos frutos previamente depelados na região da leitura.

O teor de sólidos solúveis (SS) foi determinado por meio de refratometria, utilizando refratômetro portátil com faixa de leitura de 0 a $32{ }^{\circ}$ Brix. As leituras foram feitas em amostras de suco da polpa, extraídas da homogeneização de $5 \mathrm{~g}$ de polpa, em 30 $\mathrm{mL}$ de água destilada, segundo metodologia proposta por Brasil (2005). Os resultados foram expressos em ${ }^{\circ}$ Brix.

A acidez titulável (AT) dos frutos foi determinada por meio de titulação com hidróxido de sódio $0,1 \mathrm{~N}$ do mesmo suco extraído para determinação de SST e expressa em g ácido cítrico. $100 \mathrm{~g}^{-1}$ de polpa, conforme metodologia proposta por Brasil (2005).

$\mathrm{O} \mathrm{pH}$ foi determinado por meio de peagâmetro digital (Digimed DM 20), do mesmo suco utilizado para a determinação da acidez, segundo metodologia proposta por Brasil (2005).

Os dados foram analisados por meio das análises de variância e regressão, utilizando o software SAEG (2007). Os modelos, ajustados por meio de regressão, foram escolhidos com base na significância dos coeficientes, com nível de $5 \%$ de probabilidade, pelo teste " $t$ ", no coeficiente de determinação e no potencial para explicar o fenômeno biológico. Independentemente de a interação sachês x intervalo de tempo de amostragem ser ou não significativa, optou-se pelo seu desdobramento, dado o interesse em estudo.

\section{RESULTADOS E DISCUSSÃO}

Os resultados dos halos de inibição indicam que os óleos essenciais de orégano e capim-limão foram os mais eficientes no controle do desenvolvimento dos microrganismos testados (Tabela 1).

A eficiência antimicrobiana in vitro dos sachês ativos mostra que os óleos essenciais de orégano e capim-limão foram mais eficientes no controle do desenvolvimento de microrganismos que os óleos essenciais de canela e funcho, sendo que os óleos essenciais de orégano e capim-limão não diferiram entre si no controle do crescimento dos microrganismos.

Rozwalka et al. (2008) observaram que os óleos essenciais de cravo e capim-limão inibiram totalmente o patógeno Colletotrichum gloeosporioides, e o óleo essencial de capim-limão inibiu em 100\% Glomerella cingulata até o quinto dia, apresentando redução no potencial de inibição para $62,8 \%$ no oitavo dia de conservação dos frutos de goiaba.

A cor da epiderme da manga apresentou comportamento quadrático para todos os tratamentos (Figura 1). Os frutos do tratamento-controle alcançaram coloração amarela mais rapidamente que os tratamentos Orégano e Capim-limão, sendo que o Capim-limão foi mais eficiente em manter mais baixo $o$ valor da coordenada $b^{*}$.

De acordo com Benevides et al. (2008), valores elevados de $b^{*}$ podem indicar a prevalência dos carotenoides sobre outros pigmentos, ou seja, fruto em estádio mais avançado de amadurecimento. A coordenada $b^{*}$ apresentou valor médio de 26,0 . Segundo Silva et al. (2009), valores de b* em torno de 17,0 são característicos desta cultivar, porém estes valores podem variar de acordo com a região produtora, condições climáticas e ponto de colheita. Essa variação no valores de $b^{*}$ são confirmadas no presente trabalho, pois é possível observar que, no dia de armazenamento, os frutos apresentaram valores médios de $b^{*}(29,42)$.

A firmeza de polpa apresentou comportamento quadrático (Figura 2). É possível observar queda brusca nos valores de firmeza para os tratamentos-controle e orégano, enquanto para os frutos do tratamento capim-limão esta queda foi mais suave. Rocha et al. (2001) também observaram redução na firmeza da polpa de manga 'Tommy Atkins' durante o amadurecimento pós-colheita, registrando variações de 96,1 $\mathrm{N}$ a 9,4 N. Esses decréscimos na firmeza de polpa são esperados com o amadurecimento e são caracterizados pela degradação de substâncias pécticas e de hemicelulose, e pela hidrólise do amido. 
O teor de sólidos solúveis apresentou comportamento quadrático (Figura 3), sendo que, nos frutos do tratamento capim-limão, o teor de sólidos solúveis teve tendência de estabilização, enquanto para o tratamento orégano a queda foi brusca. A queda no teor de SS no final do período de armazenamento pode estar associada ao consumo de açúcares no processo de respiração do fruto, ou a conversão de ácidos orgânicos a açúcares (CHITARRA; CHITARRA, 2005). Esse fenômeno também explica a queda nos valores de AT até os seis dias de armazenamento (Figura 4), pois os ácidos orgânicos são os primeiros a serem utilizados como substrato na respiração.

Os valores de $\mathrm{pH}$ tiveram comportamento quadrático (Figura 5), com tendência de acréscimos para todos os tratamentos, em especial o tratamento orégano, saindo de 4,4 no dia zero e 5,4 no nono dia de armazenamento.

$\mathrm{O}$ aumento do valor de AT e do $\mathrm{pH}$ no final do período de armazenamento pode ser devido à liberação dos ácidos presentes nos vacúolos que são liberados durante o amadurecimento, em consequência da descompartimentação celular. Os resultados encontrados discordam dos relatados por Subedi et al. (2007), em que houve aumento no teor de SS resultante principalmente da conversão de amido em açúcares e decréscimos nos valores de AT e pH durante o armazenamento.

Os resultados obtidos no crescimento de mesófilos aeróbios em manga mostram diferença com relação aos tratamentos avaliados e, também, em relação ao tempo de armazenamento (Figura 6). O tratamento-controle, no nono dia de armazenamento, apresentou a maior contagem, 5,67 Log UFC $\mathrm{g}^{-1}$; em contrapartida, o tratamento com sachê de óleo essencial de capim-limão obteve a menor contagem, 3,13 Log UFC $\mathrm{g}^{-1}$, para o mesmo período de avaliação, evidenciando o potencial do capim-limão em manter baixo o crescimento de mesófilos aeróbios.

A contagem de fungos filamentosos e leveduras (Figura 7) apresentou diferença com relação aos tratamentos-controle e sachê incorporado com OEs de orégano e capim-limão. É possível observar a ação do OE de capim-limão, com os menores valores em todos os tempos de análise, apresentando, no nono dia, contagem de fungos filamentosos e leveduras de apenas 3,4 UFC. ${ }^{-1}$, enquanto para frutos do tratamento-controle na mesma época de avaliação a contagem foi de 5,24 UFC. $\mathrm{g}^{-1}$.

Os resultados obtidos para mesófilos aeróbios e fungos filamentosos e leveduras apresentam o OE de capim-limão como sendo o mais eficiente no controle do crescimento desses microrganismos. Para ambos os microrganismos, mesófilos aeróbios e fungos filamentosos e leveduras, o OE de capim-limão apresentou redução de aproximadamente 2 ciclos Log em relação ao tratamento-controle.

Rodriguéz et al. (2007) desenvolveram uma embalagem de base celulósica contendo $4 \%$ de uma mistura contendo cinamaldeído enriquecido com óleo essencial de canela $(80-85 \% \mathrm{p} / \mathrm{p})$ e avaliaram sua eficiência no armazenamento de morangos. $\mathrm{O}$ filme inibiu completamente o crescimento de fungos filamentosos sobre a superfície dos frutos de morango durante sete dias, a $4{ }^{\circ} \mathrm{C}$.

De modo geral, até os seis dias de armazenamento, os tratamentos com sachês antimicrobianos, incorporados com óleos essenciais de capim-limão e orégano, apresentavam a maioria dos frutos em melhores condições de consumo que o tratamento-controle. Aos nove dias de armazenamento, o experimento foi encerrado porque os frutos de todos os tratamentos estavam em adiantado estado de decomposição, com podridão em, pelo menos, $40 \%$ de sua superfície.

A rápida senescência dos frutos é consequência do metabolismo acelerado apresentado pela manga em função de seu padrão climatérico de respiração e também pela temperatura ambiente, em torno de $25{ }^{\circ} \mathrm{C}$. Essa temperatura foi escolhida para simular as condições a que os frutos ficam expostos durante a comercialização. Como possível solução para este problema, poderia armazenar os frutos sob refrigeração ou, então, incluir dentro da embalagem um sachê com um adsorvedor de etileno, por exemplo, permanganato de potássio junto com o sachê de óleo essencial. 
TABELA 1 - Valores médios dos halos de inibição para microrganismos patogênicos na pós-colheita de manga, 'Tommy atkins'

Diâmetro de halo de inibição (mm)

\begin{tabular}{ccccc}
\cline { 2 - 4 } OE & $\begin{array}{c}\text { Alternaria } \\
\text { alternata }\end{array}$ & $\begin{array}{c}\text { Colletotrichum } \\
\text { gloeosporioides }\end{array}$ & $\begin{array}{c}\text { Lasidiplodia } \\
\text { theobromae }\end{array}$ & $\begin{array}{c}\text { Xanthomonas } \\
\text { campestris }\end{array}$ \\
\hline Orégano & $84,22^{\mathrm{a}}$ & $83,00^{\mathrm{a}}$ & $80,00^{\mathrm{a}}$ & $22,00^{\mathrm{a}}$ \\
Canela & $80,22^{\mathrm{a}}$ & $69,80^{\mathrm{a}}$ & $43,60^{\mathrm{b}}$ & $23,25^{\mathrm{a}}$ \\
Capim-limão & $82,00^{\mathrm{a}}$ & $85,79^{\mathrm{a}}$ & $79,00^{\mathrm{a}}$ & $34,00^{\mathrm{a}}$ \\
Funcho & $46,05^{\mathrm{b}}$ & $41,54^{\mathrm{b}}$ & $45,00^{\mathrm{b}}$ & $29,75^{\mathrm{a}}$ \\
\hline
\end{tabular}

*Médias seguidas da mesma letra na coluna não diferem entre si, pelo teste de Tukey $(\mathrm{p}<0,05)$.
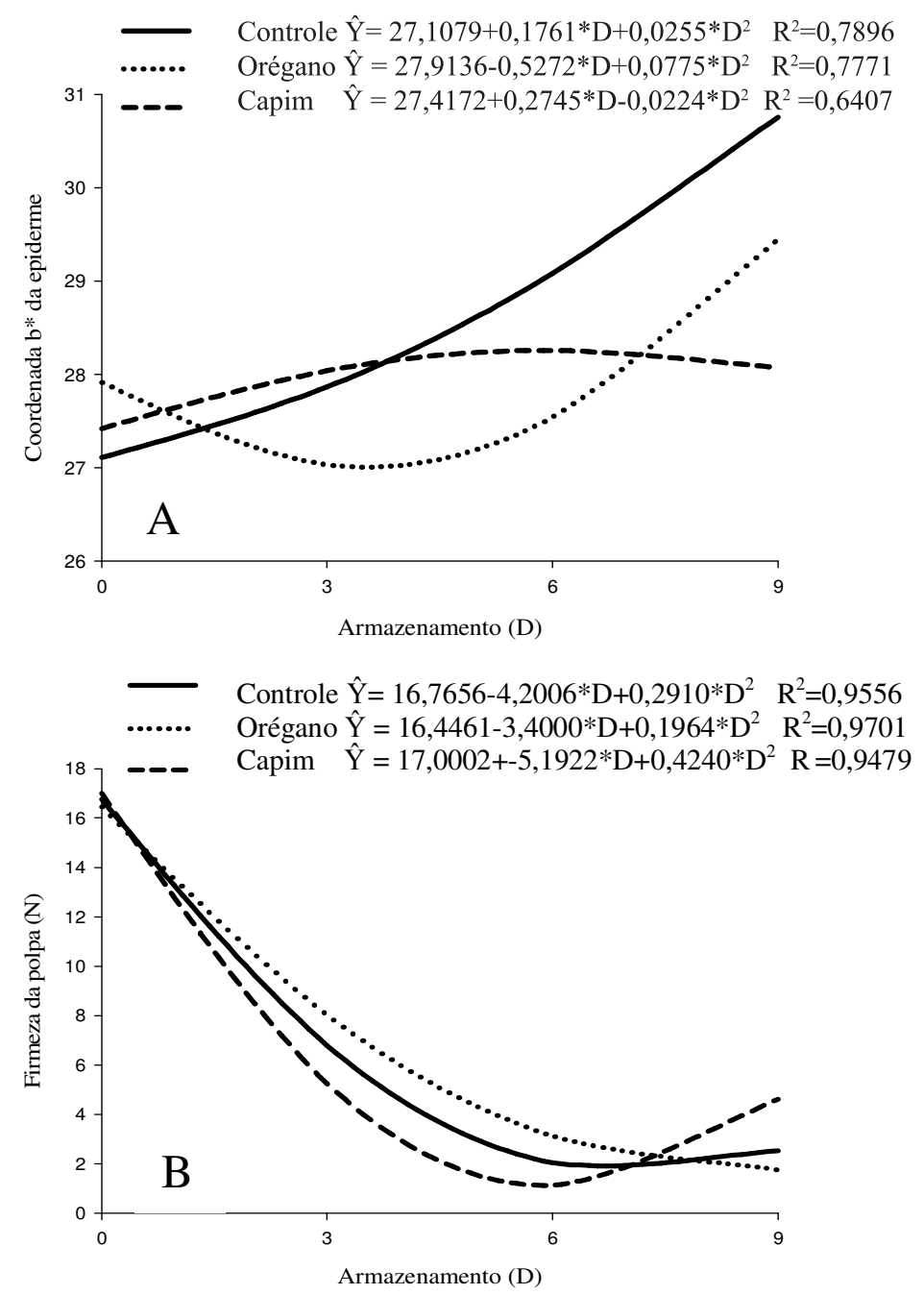


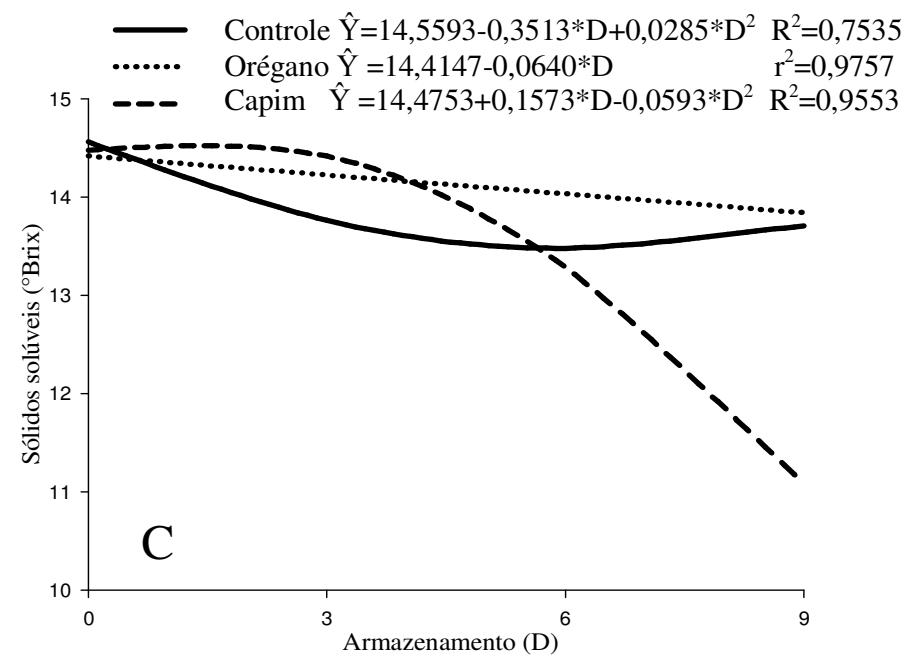

FIGURA 1- A: estimativa da cor da epiderme segundo a coordenada b* (variação da cor verde a amarela); B: estimativa da firmeza de polpa $(\mathrm{N})$, e C: estimativa do teor de sólidos solúveis (SS) de manga 'Tommy Atkins', embalada individualmente em saco de papel com sachê antimicrobiano e mantida a $25{ }^{\circ} \mathrm{C} \pm 2{ }^{\circ} \mathrm{C}$ e UR $80 \% \pm 5 \%$, por 9 dias.

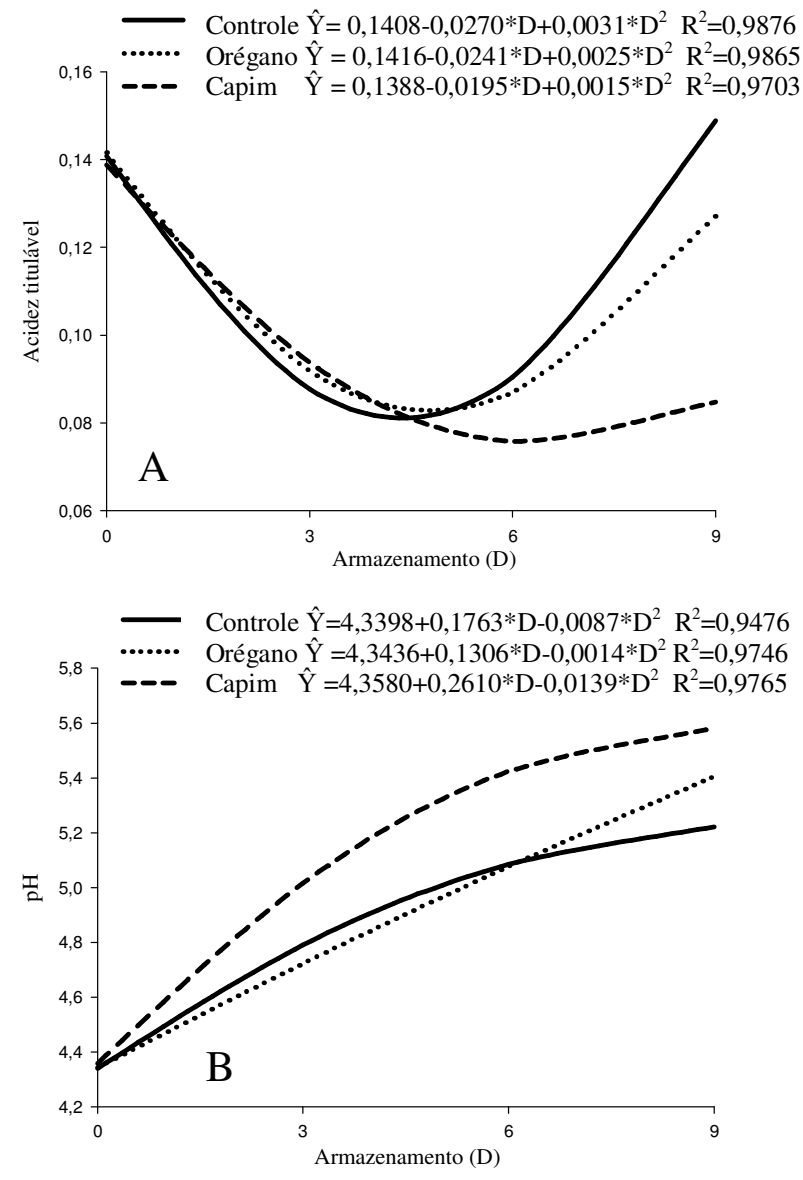

FIGURA 2- A: estimativa da acidez titulável (AT), e B: estimativa do $\mathrm{pH}$ em polpa de manga 'Tommy Atkins', embalada individualmente em saco de papel com sachê antimicrobiano e mantida a $25{ }^{\circ} \mathrm{C} \pm 2{ }^{\circ} \mathrm{C}$ e UR $80 \% \pm 5 \%$, por 9 dias. 

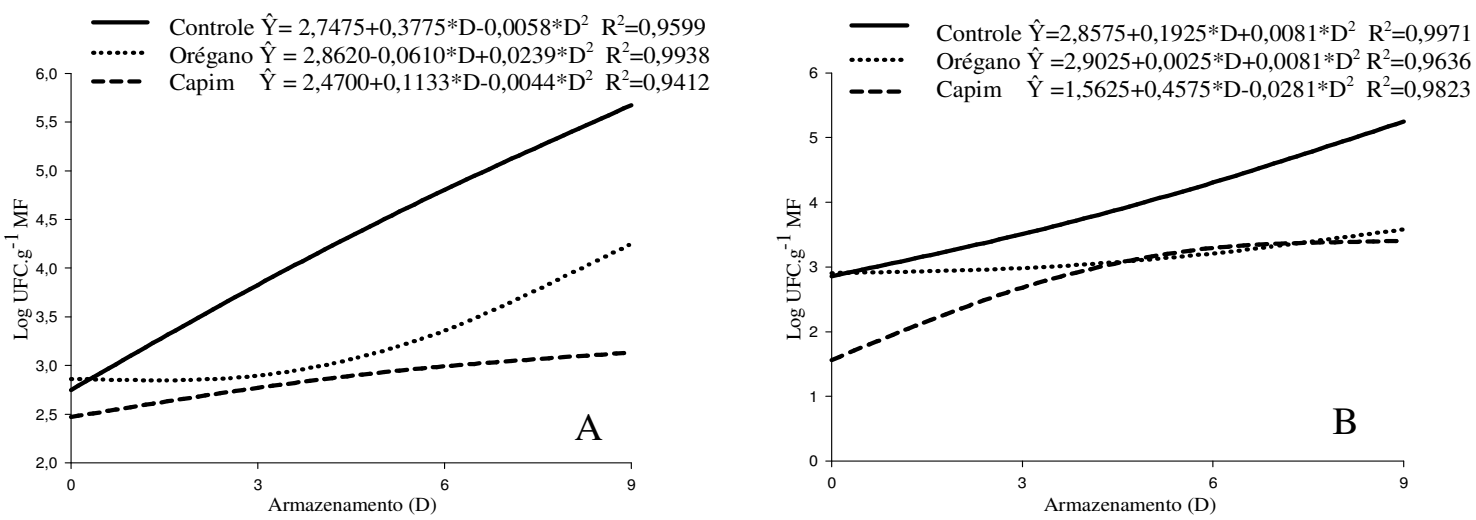

FIGURA 3- A: estimativa do crescimento de mesófilos, e B: estimativa do crescimento de fungos e leveduras em manga 'Tommy Atkins', embalada individualmente em saco de papel com sachê antimicrobiano e mantida a $25^{\circ} \mathrm{C} \pm 2{ }^{\circ} \mathrm{C}$ e UR $80 \% \pm 5 \%$, por 9 dias.

\section{CONCLUSÕES}

1. Os parâmetros cor da epiderme, firmeza de polpa, sólidos solúveis, acidez titulável e $\mathrm{pH}$ sofreram pouca influência dos óleos essenciais de orégano e capim-limão, indicando que a presença do óleo essencial não altera as características físico-químicas da polpa de manga.

2. Os sachês ativos incorporados com óleos essenciais de orégano e capim-limão apresentaram controle no crescimento dos microrganismos testados, sendo o capim-limão mais eficiente, reduzindo em aproximadamente 2 ciclos Log a contagem de mesófilos aeróbios e fungos filamentosos e leveduras em relação ao tratamento controle empregado.

\section{REFERÊNCIAS}

ALMENAR, E.; CATALA, R.; HERNANDEZMUÑOZ, P.; GAVARA, R. Optimization of an active package for wild strawberries based on the release of 2- nonanone. LWT - Food Science and Technology, Zurich, v. 42, p. 587-593. 2009.

APPENDINI, P.; HOTCHKISS, J. H. Review of antimicrobial food packaging. Innovative Food Science \& Emerging Technologies, Berlin, v. 3, p. 113-126, 2002.

BAYDAR, H.; SAGDIC, O.; ÖZKAN, G.; KARADOGAN, T. Antibacterial activity and composition of essential oils from Origanum, Thymbra and Satureja species with commercial importance in Turkey. Food Control, Guildford, v.15, n.3, p.169-172, 2004.
BENEVIDES, S. D.; RAMOS, A.M.; STRINGHETA, P. C.; CASTRO, V. C. Qualidade da manga e polpa da manga Ubá. Ciência e Tecnologia de Alimentos, Campinas, v.28, p.571-578, 2008.

BRASIL. Ministério da Saúde. Agência Nacional de Vigilância Sanitária. Métodos físico-químicos para análise de alimentos. Brasília: Ministério da Saúde, 2005. 1018 p.

CARVALHO, C. R. L.; ROSSETO, C. J.; MANTOVANI, D. M. B.; MORGANO, M. A.; CASTRO, J. V. C.; BORTOLETTO, N. Avaliação de cultivares de mangueira selecionadas pelo Instituto Agronômico de Campinas comparadas a outras de importância comercial. Revista Brasileira de Fruticultura, Jaboticabal, v. 26, n. 2, p. 264-271, 2004.

CHItARRA, M. I. F.; CHITARRA, A. B. Pós-colheita de frutos e hortaliças: fisiologia e manuseio. Lavras: Universidade Federal de Lavras, 2005. v. $1,785 \mathrm{p}$.

LEE, D. S.; HWANG, Y. I.; CHO, S. H. Developing antimicrobial packaging film for curled lettuce and soybean sprouts. Food Science and Biotechnology, London, v. 7, n. 2, p. 117-121, 1998.

LUCENA, E. M. P.; ASSIS, J. S.; ALVES, R. E.; SILVA, V. C. M.; FILHO, J. E. Alterações físicas e químicas durante o desenvolvimento de mangas 'Tommy Atkins' no Vale do São Francisco, Petrolina - PE. Revista Brasileira de Fruticultura, Jaboticabal, v. 29, n. 1, p. 96-101, 2007. 
ROCHA, R. H. C.; MENEZES, J. B.; MORAIS, E. A. DE; SILVA, G. G. DA; AMBRÓSIO, M. M. DE Q.; ALVEZ, M. Z. Uso do índice de degradação de amido na determinação da maturidade da manga 'Tommy Atkins'. Revista Brasileira de Fruticultura, Jaboticabal, v. 23, n. 2, p. 302-305, 2001.

RODRÍGUEZ, A.; BATLLE, R.; NERÍN, C. The use of natural essential oils as antimicrobial solutionsin paper packaging. Part II. Progress in Organic Coatings, Lausanne, v.60, p. 33-38, 2007.

ROZWALKA, L. C.; LIMA, M. L. R. Z. C.; MIO, L. L. M.; NAKASHIMA, T. Extratos, decoctos e óleos essenciais de plantas medicinais e aromáticas na inibição de Glomerella cingulata e Colletotrichum gloeosporioides de frutos de goiaba. Ciência Rural, Santa Maria, v. 38, n. 2, p. 301-307, 2008.
SILVA, D. F. P.; SIQUEIRA, D. L.; PEREIRA, C. S.; SALOMÃO, L. C. C.; STRUIVING, T. B. Caracterização de frutos de 15 cultivares de mangueira na Zona da Mata Mineira. Revista Ceres, Viçosa, MG, v. 56, n.6, p. 783-789, 2009.

SOUZA, E. L.; STAMFORD, T. L. M.; LIMA, E. O.; TRAJANO, V. N. Effectiveness of Origanum vulgare $\mathrm{L}$. essential oil to inhibit the growth of food spoiling yeasts. Food Control, Guildford, v.18, n. 5, p.409-413, 2007.

SUBEDI, P. P.; WALSH, K. B.; OWENS, G. Prediction of mango eating quality at harvest using short-wave near infrared spectrometry. Postharvest Biology and Technology, Amsterdam, v. 43, n. 3, p. 326-334, 2007.

ZIVANOVIC, S.; CHI, S.; DRAUGHON, A. F. Antimicrobial activity of chitosan films enriched with essential oils. Journal of Food Science, Chicago, v. 70, n. 1, p. 45-51. 2005. 